\title{
TESTE RÁPIDO DE IMERSÃo FOLIAR DE Euphorbia heterophylla PARA Confirmação de Resistência A Herbicidas InIbIdores da Protox E DA ALS $\mathbf{S}^{1}$
}

\author{
Quick Test of Foliar Immersion of Euphorbia heterophylla to Confirm Resistance to PPO and \\ ALS-Inhibiting Herbicides
}

TREZZI, M.M. ${ }^{2}$, XAVIER, E. ${ }^{3}$, VIDAL, R.A. ${ }^{4}$, PORTES, E.D.S. ${ }^{5}$, VOTTRI, M. ${ }^{6}$ e LAMEGO, F.P. ${ }^{7}$

\begin{abstract}
RESUMO - Os testes utilizados para identificação de biótipos resistentes de plantas daninhas aos herbicidas variam segundo o tempo de execução e o grau de complexidade, sendo necessário determinar a eficácia de métodos rápidos e de simples execução. Foram realizados dois ensaios, simultaneamente, em laboratório, em delineamento completamente casualizado, empregando a imersão de folhas de E. heterophylla suscetiveis e resistentes a inibidores da ALS e da PROTOX em solução herbicida. A parte aérea das plantas foi submersa em solução herbicida com diferentes concentrações de imazethapyr, imazapyr e nicosulfuron (inibidores da ALS) e fomesafen, lactofen e carfentrazone (inibidores da PROTOX). O controle do biótipo suscetivel foi crescente com o decorrer do tempo, e as doses comerciais dos inibidores da ALS e da PROTOX testados apresentaram controle eficiente. O biótipo resistente mostrou diferentes niveis de resistência em função do herbicida testado e da variável considerada. Os resultados encontrados respaldam a técnica da imersão foliar como adequada para a discriminação de biótipos suscetiveis ou com resistência múltipla a inibidores da ALS e PROTOX. A técnica demonstrou ser rápida o suficiente para a detecção precoce da resistência, o que possibilitaria a adoção de medidas de contenção ainda na mesma safra.
\end{abstract}

Palavras-chave: leiteira, curvas de dose-resposta, resistência múltipla, resistência de plantas daninhas a herbicidas.

\begin{abstract}
Tests to verify resistance $(R)$ vary according to time of execution and degree of complexity, and the efficacy of rapid and simple methods to confirm weed resistance to herbicides must be determined. Two experiments were simultaneously carried out in a completely randomized design, under laboratory conditions, by submerging leaves of $\boldsymbol{E}$. heterophylla susceptible and with multiple resistance to $A L S$-and $P P O$ inhibitors in herbicide solutions. The aerial part of the plants was submerged in herbicide solution at different concentrations of imazethapyr, imazapyr, and nicosulfuron (ALS inhibitors), and fomesafen, lactofen and carfentrazone (PPO inhibitors). S biotype control increased with time and commercial ratings of ALS and PROTOX inhibitors had an effective control. The $R$ biotype showed different levels of resistance as a function of the herbicide and variable evaluated. The results confirm that leafimmersion technique is adequate to discriminate $S$ biotypes from those with multiple resistance to ALS and PPO inhibitors. The technique proved to be quick enough for early detection of resistance, allowing the adoption of measures to deal with resistance during the same season.
\end{abstract}

Keywords: wild poinsettia, dose-response curves, multiple resistance, weed resistance to herbicides

1 Recebido para publicação em 16.9.2010 e aprovado em 8.2.2011.

2 Professor, Dr., Curso de Agronomia, Universidade Tecnológica Federal do Paraná, Campus Pato Branco, Via do Conhecimento, km 01, Caixa Postal 571, 85503-390 Pato Branco-PR, <trezzi@utfpr.edu.br>; ${ }^{3}$ Acadêmica do Curso de Agronomia da UTFPR; ${ }^{4}$ Professor, Ph.D., Universidade Federal do Rio Grande do Sul, Porto Alegre-RS, ${ }^{5}$ Professor, M.Sc., Curso de Agronomia SEI/FAI, Itapiranga-SC; ${ }^{6}$ Eng-o-Agro ${ }^{-}$, UTFPR; ${ }^{7}$ Professor, Ph.D., Universidade Federal de Santa Maria/CESNOR, Frederico Westphalen-RS.

Planta Daninha, Viçosa-MG, v. 29, n. 4, p. 901-912, 2011 


\section{INTRODUÇÃO}

A adoção do controle químico, através do uso de herbicidas, representou um avanço nas técnicas de manejo de plantas daninhas (Vidal et al., 2006). No entanto, a resistência de plantas daninhas a herbicidas - um efeito colateral do seu uso - tem se tornado cada vez mais importante após anos consecutivos do uso desses produtos. Atualmente, existem mais de 360 biótipos resistentes (R) a herbicidas no mundo, envolvendo 197 espécies daninhas (Heap, 2011).

A resistência de plantas daninhas aos herbicidas é uma resposta evolutiva dessas espécies de plantas às práticas agrícolas. O desenvolvimento da resistência é influenciado por fatores genéticos, como a frequência inicial do alelo resistente e a dominância do gene determinante da resistência; por fatores bioecológicos, que incluem ciclo de vida, produção de sementes, número de gerações de produção de sementes, etc.; e por fatores agronômicos, como o período residual dos herbicidas, sua frequência de uso, a eficiência de controle e a dose utilizada (Christoffoleti \& Lopes-Ovejero, 2008).

O uso repetitivo de um herbicida ou de herbicidas com o mesmo mecanismo de ação exerce alta pressão de seleção, o que reduz ou elimina indivíduos suscetiveis (S) e acaba por aumentar o número de indivíduos tolerantes e a manifestação de biótipos $\mathrm{R}$ que, provavelmente, já existiam na população, mas em frequência mais baixa (Christoffoleti \& Lopes Ovejero, 2008)

Entre as principais consequências da resistência de plantas daninhas a herbicidas estão a limitação ou inviabilização do uso de determinados herbicidas, perda de áreas para semeadura, perda de rendimento e qualidade dos produtos das culturas agrícolas, necessidade de reaplicação de herbicidas e mudanças em sistemas de produção. A grande preocupação com esse tema decorre, também, do aumento nos custos de controle de plantas daninhas, principalmente em razão do limitado - ou em alguns casos inexistente - número de herbicidas alternativos para o controle dos biótipos R.

No mundo todo, são encontrados somente nove biótipos de plantas daninhas resistentes aos inibidores da PROTOX, em quatro espécies diferentes: Ambrosia artemisiifolia (EUA), Amaranthus tuberculatus (EUA), Amaranthus quitensis (Bolivia) e Euphorbia heterophylla (Brasil) (Heap, 2011). Os biótipos de E. heterophylla referidos são resistentes tanto a inibidores da ALS quanto a inibidores da PROTOX e foram detectados em lavouras da região sudoeste do Paraná (Trezzi et al., 2005). Quando o herbicida fomesafen foi aplicado na parte aérea desses biótipos, houve menor nível de controle do biótipo $R$, comparativamente ao S; no entanto, quando esse herbicida foi aplicado no solo, não houve diferença significativa entre os biótipos, sugerindo que o mecanismo está relacionado à absorção dos herbicidas inibidores da PROTOX pela parte aérea das plantas de E. heterophylla (Trezzi et al., 2009).

Diagnosticar a resistência em uma população de plantas daninhas de forma rápida, eficaz e precisa ajuda a prevenir a disseminação de sementes $\mathrm{R}$ na área, evitando problemas futuros e permitindo a tomada de decisão correta e em tempo hábil para o manejo de plantas daninhas na área (Vidal et al., 2006). Aliada à identificação da resistência, a detecção precoce da população $R$ ainda nos estádios iniciais da colonização da área, por meio de monitoramento constante das lavouras, provavelmente prevenirá a disseminação da resistência na área, evitando o incremento do banco de sementes do solo e, consequentemente, reduzindo os custos e o impacto do manejo desses biótipos (Vidal et al., 2006).

Mundialmente, têm sido desenvolvidos diversos métodos para diagnosticar a resistência em várias espécies daninhas. Alguns métodos mostram-se mais vantajosos para determinadas situações em que, por exemplo, há limitação de recursos; já outros são mais sofisticados e requerem mão de obra especializada. O importante é que sejam precisos e garantam a certificação de resistência ou não a determinado grupo herbicida (Vidal \& Merotto Júnior, 1999).

Os mais comuns são os bioensaios em casa de vegetação, por meio de curvas de doseresposta (Seefeldt et al., 1995). São utilizados também bioensaios com germinação de sementes (Carvalho et al., 2004; Concenço et al, 2008), ensaios com partes de plantas (Kim et al., 2000; Boutsalis, 2001), quantificação de 
enzimas (Monquero \& Christoffoleti, 2001b), germinação de pólen (Richter \& Powles, 1993), fluorescência da clorofila (Norsworthy et al., 1998) e avaliação do RNA ou DNA da enzima do mecanismo de ação (Saari et al., 1994).

Entre as desvantagens dos métodos citados está o elevado tempo para detecção de resistência, caso haja necessidade de desenvolvimento da planta, sementes ou pólen para o diagnóstico, ou, ainda, o custo elevado das análises e a necessidade de mão de obra especializada, especialmente para as análises em laboratório (Vidal et al., 2006).

Um método diagnóstico da resistência de plantas daninhas em laboratório eficiente, rápido e fácil de executar, utilizando plântulas imersas em solução herbicida, foi desenvolvido por Portes (2005), o qual foi denominado UFRGS Super-Quick Test for Herbicide Resistance (Vidal et al., 2005). Após imersão na solução herbicida, as plântulas são colocadas em água, havendo necessidade de apenas quatro dias para a identificação de sintomas em plantas suscetiveis aos inibidores de ALS e ACCase (Portes, 2005).

O objetivo deste trabalho foi avaliar a eficiência do método diagnóstico de resistência através da imersão da parte aérea de plântulas de Euphorbia heterophylla em soluções de herbicidas inibidores da PROTOX e da ALS.

\section{MATERIAL E MÉTODOS}

O experimento foi conduzido no Laboratório de Sementes do Curso de Agronomia da Universidade Tecnológica Federal do Paraná, campus Pato Branco. Foram utilizados biótipos de Euphorbia heterophylla (EPHHL) suscetiveis (S) e com resistência múltipla aos herbicidas inibidores da ALS e PROTOX (R).

Dois ensaios foram realizados simultaneamente, em laboratório, em delineamento completamente casualizado, com cinco repetições. Os tratamentos consistiram da submersão da parte aérea das plantas na solução herbicida, utilizando metodologia semelhante à adotada por Portes (2005). O primeiro ensaio consistiu do uso de curvas de dose-resposta, utilizando-se um biótipo S de EPHHL, adquirido na empresa Agrocosmos, enquanto o segundo ensaio foi realizado com biótipo de EPHHL resistente a ALS e PROTOX, conforme previamente identificado por Trezzi et al. (2005). Para a variável controle, os tratamentos de ambos os ensaios foram arranjados em um fatorial $6 \times 8 \times 3$, sendo o fator A constituído pelos herbicidas inibidores da PROTOX (fomesafen, lactofen e carfentrazone) e da ALS (imazethapyr, imazapyr e nicosulfuron); o fator $\mathrm{B}$, pelas concentrações ajustadas mediante ensaios preliminares; e o fator C, pelas épocas de avaliação (1, 3 e 5 dias após o tratamento para os inibidores da PROTOX e 4, 7 e 10 dias para os inibidores de ALS). Para as variáveis matéria seca e verde, foi utilizado o fatorial $6 \times 8$, pois elas foram avaliadas apenas no término do experimento.

As plântulas de EPHHL utilizadas nos ensaios de dose-resposta, em laboratório, foram obtidas em casa de vegetação, pela semeadura de sementes de EPHHL R e S em bandejas de 128 células, contendo substrato organomineral. Assim que as plântulas atingiram o estádio de duas folhas verdadeiras, foram retiradas do substrato, sendo suas raízes lavadas em água corrente, e armazenadas em frasco com água destilada por no máximo duas horas, até o uso nos ensaios de doseresposta.

$\mathrm{O}$ ajuste de doses foi realizado de forma que se obtivesse a maior amplitude acima e abaixo da concentração utilizada comercialmente, possibilitando verificar resultados dentro da faixa de resposta de cada biótipo. Para isso, utilizou-se a concentração comercial como dose máxima para o biótipo $\mathrm{S}$ e, para o biótipo $\mathrm{R}$, doses substancialmente acima da máxima referida anteriormente, visando ao controle total das plantas.

Para o ensaio com o biótipo S, as oito proporções da dose comercial (x) utilizadas foram $0 \mathrm{x}, 0,09 \mathrm{x}, 0,13 \mathrm{x}, 0,20 \mathrm{x}, 0,30 \mathrm{x}, 0,44 \mathrm{x}, 0,67 \mathrm{x}$ e $1 \mathrm{x}$, correspondentes às concentrações de 0 , $5,2,7,9,11,85,17,7,26,6,40$ e $60 \mathrm{mg} \mathrm{L}^{-1} \mathrm{de}$ carfentrazone; de 0, 109,7, 164,6, 246,9, 370,4, $555,5,833,33$ e $1.250 \mathrm{mg} \mathrm{L}^{-1}$ de fomesafen; de $0,105,3,158,1,237,355,5,533,3,800$ e $1.200 \mathrm{mg} \mathrm{L}^{-1}$ de lactofen; de 0, 219,5, 329,2, $493,8,740,7,1.111,1,1.666,6$ e $2.500 \mathrm{mg} \mathrm{L}^{-1}$ de imazapyr; de 0, 26,3, 39,5, 59,3, 88,9, 133,3, 200 e $300 \mathrm{mg} \mathrm{L}^{-1}$ de nicosulfuron; e de 0, 43,9, $65,8,98,7,148,1,222,2,333,3$ e $500 \mathrm{mg} \mathrm{L}^{-1} \mathrm{de}$ imazethapyr. 
Para o ensaio com o biótipo $\mathrm{R}$, as oito proporções da dose comercial utilizadas para os inibidores de PROTOX foram $0 \mathrm{x}, 1 \mathrm{x}, 1,5 \mathrm{x}, 2,17 \mathrm{x}$, $3,37 \mathrm{x}, 5,06 \mathrm{x}, 7,58 \mathrm{x}$ e $11,39 \mathrm{x}$, correspondentes às concentrações de $0,60,90,135,202,5$, 303,7, 455,6 e 683,4 $\mathrm{mg} \mathrm{L}^{-1}$ de carfentrazone; $0,1.250,1.875,2.812,5,4.218,7,6.328,1$, 9.492,2 e $14.238,3 \mathrm{mg} \mathrm{L}^{-1}$ de fomesafen; de 0, $1.200,1.800,2.700,4.050,6.075,9.112,5 \mathrm{e}$ $13.688,8 \mathrm{mg} \mathrm{L}^{-1}$ de lactofen; no caso dos inibidores da ALS, foram 0x, 0,30x, 0,44x, $0,67 \mathrm{x}, 1 \mathrm{x}, 1,50 \mathrm{x}, 2,25 \mathrm{x}, 3,38 \mathrm{x}$, correspondentes às concentrações de $0,740,7,1.111,1$, $1.666,6,2.500,3.750,5.625$ e $8.437,5 \mathrm{mg} \mathrm{L}^{-1}$ de imazapyr; 0, 88,9, 133,3, 200, 300, 450, 675 e $1.012,5 \mathrm{mg} \mathrm{L}^{-1}$ de nicosulfuron; e $0,148,15$, $222,2,333,3,500,750,1.125$ e $1.687 \mathrm{mg} \mathrm{L}^{-1} \mathrm{de}$ imazethapyr.

Em laboratório, as plântulas tiveram sua parte aérea mergulhada em solução herbicida por dois segundos, sendo posteriormente transferidas para frascos com $20 \mathrm{~mL}$ de capacidade, preenchidos com água destilada, na qual apenas o sistema radicular das plântulas ficou imerso. Utilizou-se apenas uma plântula por frasco. Os frascos foram mantidos em câmara de crescimento, com fotoperíodo de 12 horas e temperatura de $26^{\circ} \mathrm{C}$.

Para os herbicidas inibidores da PROTOX, os danos às plântulas foram avaliados visualmente 1,3 e 5 dias após a imersão (DAI), de acordo com a escala para avaliação de controle e injúria (Frans et al., 1986); aos 5 DAI também foi determinada a massa verde (MV) de plântulas, em balança com precisão de $0,1 \mathrm{mg}$. Quanto aos herbicidas inibidores da enzima ALS, avaliaram-se os sintomas visuais aos 4, 7 e 10 DAI e determinou-se a MV aos 10 DAI (Portes, 2005). Após a determinação de MV, as plântulas foram desidratadas em estufa a $60^{\circ} \mathrm{C}$ até atingirem peso constante, sendo determinada a massa seca (MS).

Os dados foram submetidos à análise de variância, pelo teste $\mathrm{F}(\mathrm{P}<0,05)$. As relações entre variáveis dependentes e as concentrações de herbicidas foram ajustadas através de modelos de regressão não linear, empregandose o modelo logístico de quatro parâmetros e, no caso de não haver ajustamento a este modelo, priorizando, em ordem decrescente, $o$ modelo logístico de três parâmetros e os sigmoidais, de quatro e três parâmetros. $\mathrm{O}$ ajuste aos modelos foi feito com auxílio do aplicativo Sigmaplot 10.0. A partir desses modelos foram obtidos os $\mathrm{I}_{50}$ (dose necessária para produzir $50 \%$ de injúria ou reduzir em $50 \%$ o desenvolvimento das plantas). Os fatores de resistência (FRs) foram calculados por meio do quociente entre os parâmetros $\mathrm{I}_{50}$ dos biótipos R e S.

As médias entre biótipos foram comparadas utilizando-se o teste da diferença mínima significativa (DMS) a $5 \%$ de probabilidade.

\section{RESULTADOS E DISCUSSÃO}

Nos ensaios com os biótipos R e S, na análise da variância da variável controle por avaliação visual, houve interação significativa entre herbicida, dose e os períodos de avaliação. Em ambos os ensaios ocorreu interação significativa entre dose e herbicida, para as variáveis matéria verde e seca.

Em geral, nesses experimentos, observouse aumento do controle dos biótipos $\mathrm{R}$ e S com a elevação da dose de herbicidas de ambos os mecanismos de ação testados (inibidores de PROTOX e ALS), com valores significativamente maiores para o biótipo S de Euphorbia heterophylla (EPHHL) do que para o R (Figuras 1 e 2). Também, foram observados aumentos nos niveis de controle para cada biótipo com o decorrer do tempo, tanto para os herbicidas inibidores da ALS quanto para os inibidores da PROTOX (Figuras 1 e 2).

Os niveis de controle do biótipo $\mathrm{S}$ obtidos com a dose comercial de inibidores da ALS (1x), nos três períodos de avaliação (4, 7 e $10 \mathrm{DAI})$, foram de 44, 72 e $96 \%$ para o imazethapyr; de 46,80 e $97 \%$ para o imazapyr; e de 44,68 e $98 \%$ para o nicosulfuron, respectivamente (Figura 1D, E, F). Nessa concentração, os niveis de controle do biótipo $\mathrm{R}$, nos mesmos períodos de avaliação supracitados, foram de 5,10 e $16 \%$ para o imazethapyr, de 4,9 e $17 \%$ para o imazapyr e de 3, 5 e $14 \%$ para o nicosulfuron (Figura 1A, B, C). Ou seja, a dose comercial foi altamente discriminadora do comportamento diferencial entre biótipos, pois resultou em elevada eficiência de controle do biótipo $\mathrm{S}$ e niveis de controle muito baixos do biótipo $R$. Mesmo com a utilização de concentrações aproximadamente 3,4 vezes superiores à normalmente empregada, os niveis máximos 
de controle do biótipo R, aos 10 DAI, atingiram 54,52 e $48 \%$, respectivamente para imazapyr, nicosulfuron e imazethapyr (Figura 1C).

Também empregando a metodologia de imersão da parte aérea em solução herbicida, Portes (2005) usou concentrações distintas de imazapyr em biótipos $\mathrm{R}$ e $\mathrm{S}$ de Bidens pilosa e obteve niveis de controle aos 7 DAI de 64 e $6 \%$, respectivamente, com a concentração de $2.500 \mathrm{mg} \mathrm{L}^{-1}$. Neste experimento, é relatada a detecção apenas aos $7 \mathrm{DAI}$, porém não foram documentadas avaliações antes desse período para demonstrar se seriam capazes de detectar diferenças entre os biótipos $\mathrm{R}$ e $\mathrm{S}$.

A imersão das folhas do biótipo $\mathrm{S}$ em concentração comercial (1X) de herbicidas
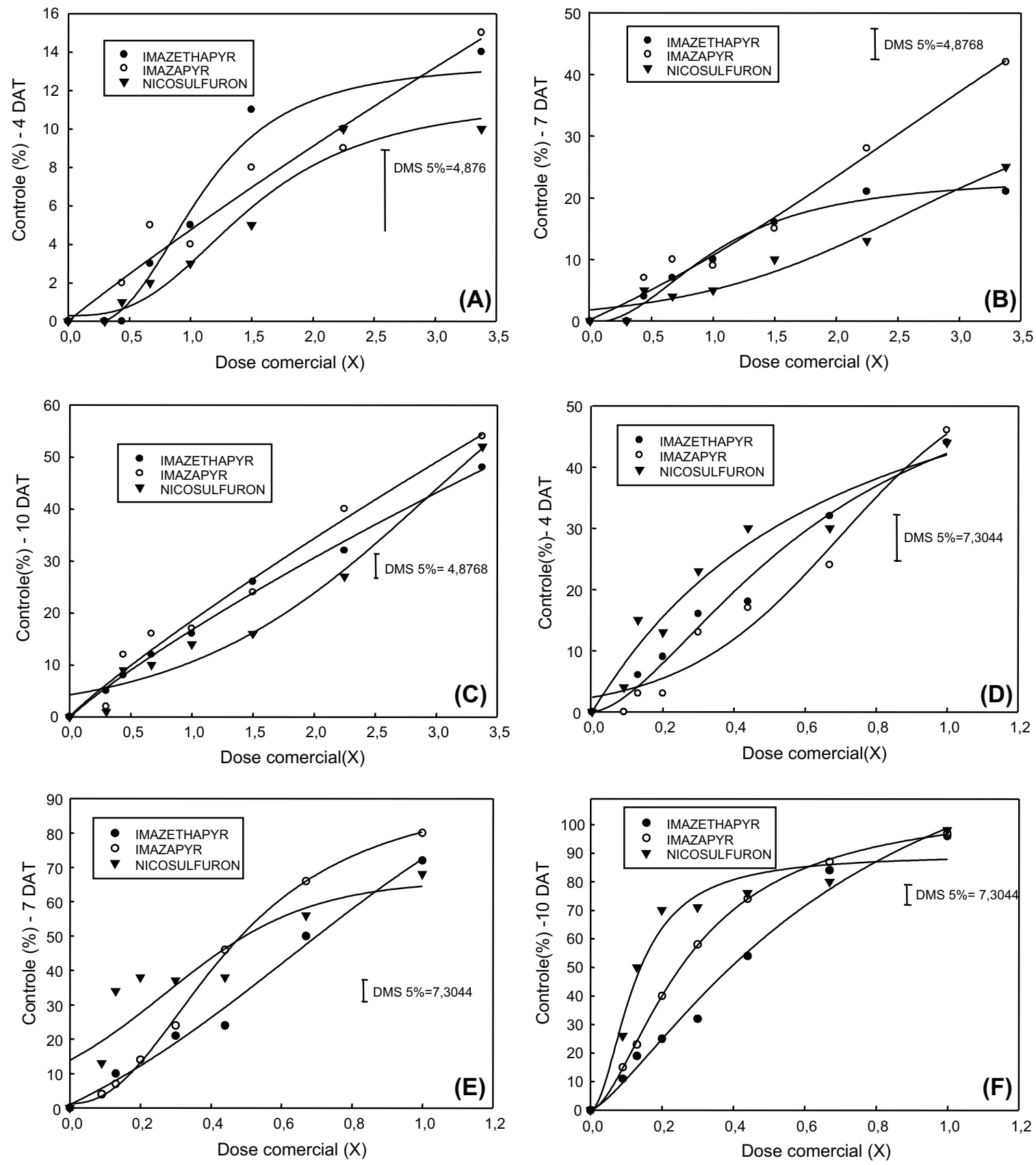

Figura 1 - Controle (\%) de plantas de E. heterophylla dos biótipos resistente, aos 4 (A), 7 (B) e 10 (C) dias após imersão (DAI), e suscetível, aos 4 (D), 7 (E) e 10 (F) dias após imersão (DAI), em função das doses dos inibidores de ALS. 
inibidores de PROTOX resultou em niveis de controle de 46, 90 e $96 \%$ para fomesafen, de 23, 78 e $100 \%$ para lactofen e de 23, 62 e 99\% para carfentrazone, respectivamente, para as avaliações aos 1, 3 e 5 DAT (Figura 2D, E, F). Nessa concentração, os niveis de controle do biótipo $R$, nos mesmos períodos de
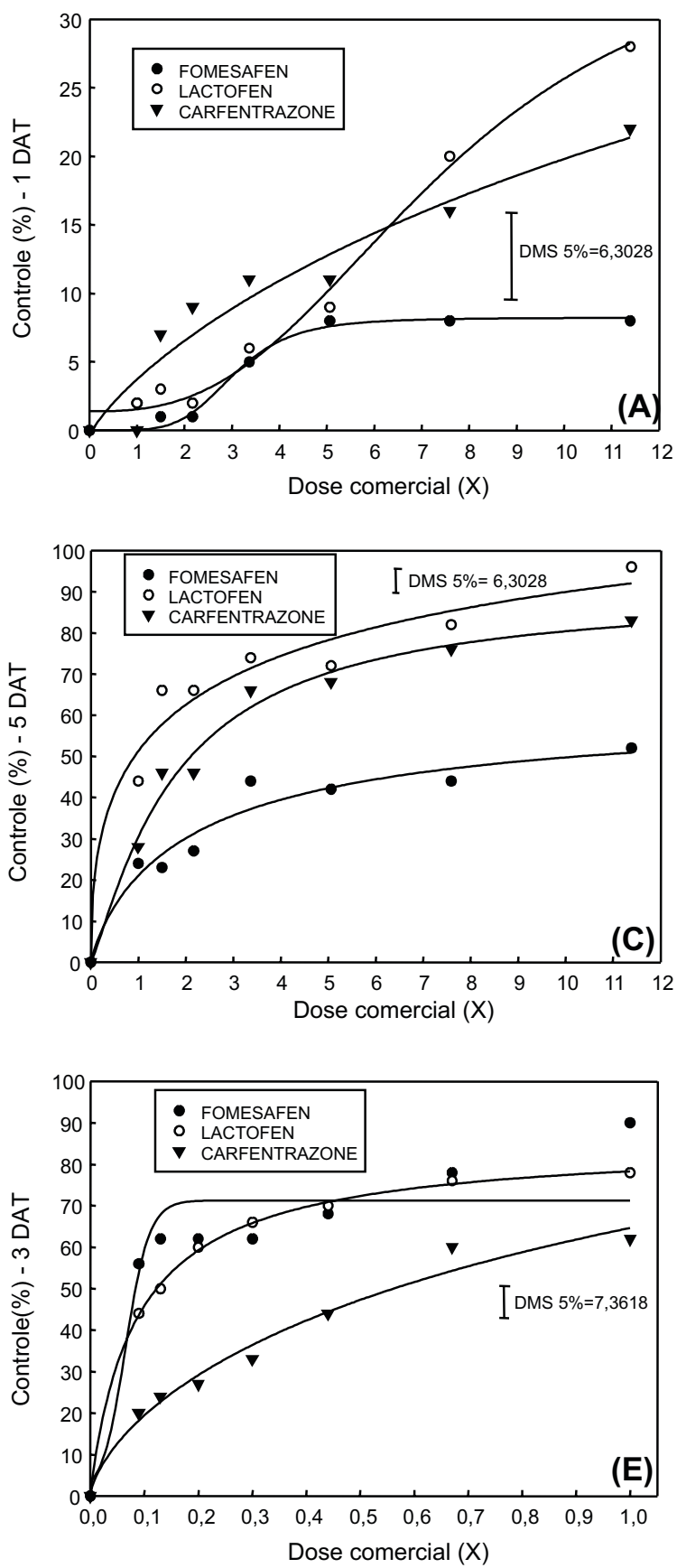

avaliação referidos, foram de 2, 10 e $24 \%$ para o fomesafen, de 2, 23 e 44\% para o lactofen e de 0,10 e $28 \%$ para o carfentrazone (Figura 2A, B, C). Assim, comprova-se que a dose comercial $(1 \mathrm{x})$ de inibidores da PROTOX também apresenta elevada capacidade discriminadora de niveis de controle entre biótipos $\mathrm{R}$ e $\mathrm{S}$,
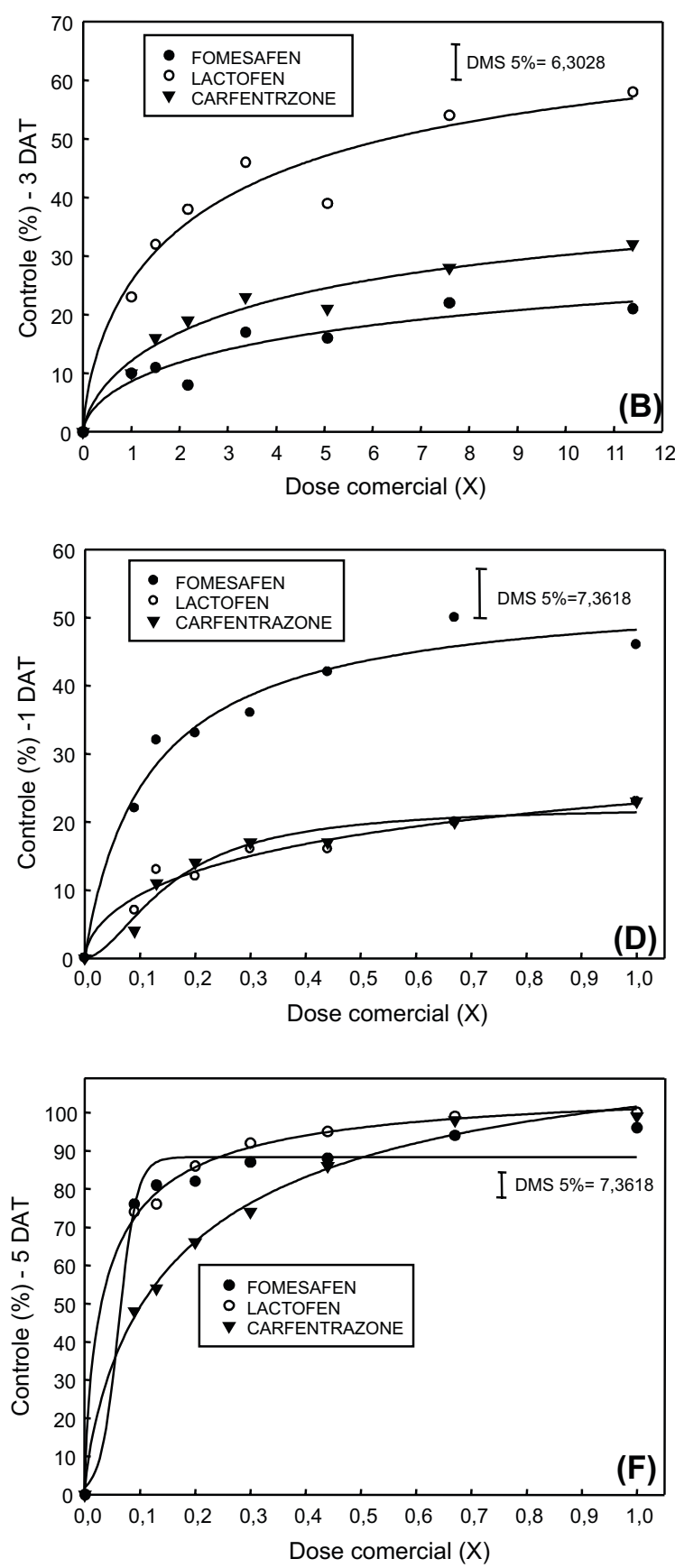

Figura 2 - Controle (\%) de plantas de E. heterophylla dos biótipos resistente, aos 1 (A), 3 (B) e 5 (C) dias após imersão (DAI), e suscetível, aos 1 (D), 3 (E) e 5 (F) dias após imersão (DAI), em função das doses dos inibidores de PROTOX. 
assim como fora observado para herbicidas inibidores da ALS.

Em casa de vegetação, comparando o mesmo biótipo R utilizado no presente experimento com o biótipo S, Trezzi et al. (2009) constataram que a dose de fomesafen recomendada pelo fabricante em pós-emergência resultou em controle do biótipo $\mathrm{S}$ de $80 \%$ e de menos de $30 \%$ do biótipo $R$, aos 14 e 21 dias após a aplicação do herbicida. Esses valores foram bastante similares aos obtidos com doses comerciais no presente experimento, mas com tempo necessário para gerar os sintomas nas plantas superior ao do ensaio de imersão da parte aérea das plantas. A utilização do ensaio em laboratório, em condições controladas, permite uniformizar melhor o ambiente para o desenvolvimento das plantas e garante condições mais adequadas para o seu desenvolvimento após o contato com o herbicida, o que permite otimizar a atividade fisiológica deste. Em condição não controlada, ao contrário, temperatura, radiação solar, umidade relativa, etc. podem estar fora da faixa adequada para maximizar a ação dos herbicidas.

Embora as maiores diferenças entre biótipo $\mathrm{S}$ e $\mathrm{R}$ a inibidores de ALS tenham sido constatadas aos 10 DAT, é importante salientar que foi possivel distinguir diferenças visuais de controle entre o biótipo suscetivel e o resistente após imersão foliar com a concentração comercial de inibidores da ALS (1X) já aos 4 DAT (Figura 1). Para os inibidores da PROTOX, a técnica da imersão foliar permitiu a distinção entre o biótipo $\mathrm{R}$ e o $\mathrm{S}$ já no primeiro dia de utilização da dose comercial (1X). A julgar pelos niveis de controle de inibidores da ALS no biótipo S obtidos nessa avaliação (44 a 46\%), é muito provável que a detecção de diferenças entre os biótipos possa ter sido efetuada mais precocemente do que 4 DAT, porém não foram realizadas avaliações em intervalos menores de tempo.

A detecção precoce de diferenças entre biótipos R e S é um dos principais objetivos dos bioensaios, pois métodos rápidos proporcionam a adoção de medidas de controle ainda na safra em que foi constatado o problema. A morosidade da diagnose acarreta prejuízos à cultura devido à interferência das plantas daninhas $\mathrm{R}$ que não são controladas e devido ao custo com herbicidas aplicados erroneamente na área (Boutsalis, 2001). Além disso, as plantas R que não são adequadamente controladas na safra servirão para a multiplicação de sementes e alimentação do banco de sementes nas áreas, contribuindo para aumentar o problema nas safras seguintes. $\mathrm{O}$ bioensaio com imersão de folhas permitiria a realização da coleta cuidadosa de plântulas de EPHHL no campo e a aplicação do método em laboratório, com a utilização de concentrações discriminadoras de biótipos R e S. Esse processo ocorreria em um tempo considerado muito curto, comparativamente a outros ensaios com aplicação de herbicidas em plantas obtidas a partir de sementes coletadas em campo.

No caso do bioensaio proposto por Boutsalis (2001), chamado de Syngenta Quick Test (QT), que analisa estacas de mudas das daninhas suspeitas de resistência sem utilizar sementes, o tempo do resultado do teste estará disponivel em um período de 40 dias da coleta das plantas, o que pode ser considerado um período longo, levando-se em conta que em um mês as plantas já estariam em um estádio avançado de desenvolvimento, e isso complicaria ainda mais o controle delas. No método de laboratório proposto por Concenço et al. (2008), que consistiu em análise de resistência pela germinação de sementes através do teste-padrão, o tempo necessário para obter os primeiros resultados é de sete dias. Para confirmação da resistência são necessários 14 dias, tempo considerado curto para a detecção, mas isso não permite o teste rápido com plântulas coletadas na própria área suspeita.

Comparando os niveis de controle de concentrações de inibidores de PROTOX utilizados no biótipo $R$, cerca de 11 vezes a máxima utilizada para o $\mathrm{S}$, constata-se que somente os niveis de controle obtidos por lactofen equipararam-se ao efeito exercido pela dose comercialmente utilizada no biótipo S (Figura 2C, F). Os niveis de controle aos 5 DAI do biótipo R proporcionados por fomesafen e carfentrazone em concentração 11 vezes superior foram inferiores aos obtidos com o controle do biótipo $\mathrm{S}$ pela dose comercial (1X) desses herbicidas (Figura 3C, F).

No biótipo $\mathrm{S}$, todos os herbicidas inibidores da PROTOX apresentaram controle satisfatório, inclusive em concentrações menores do que as recomendadas em campo. Em 

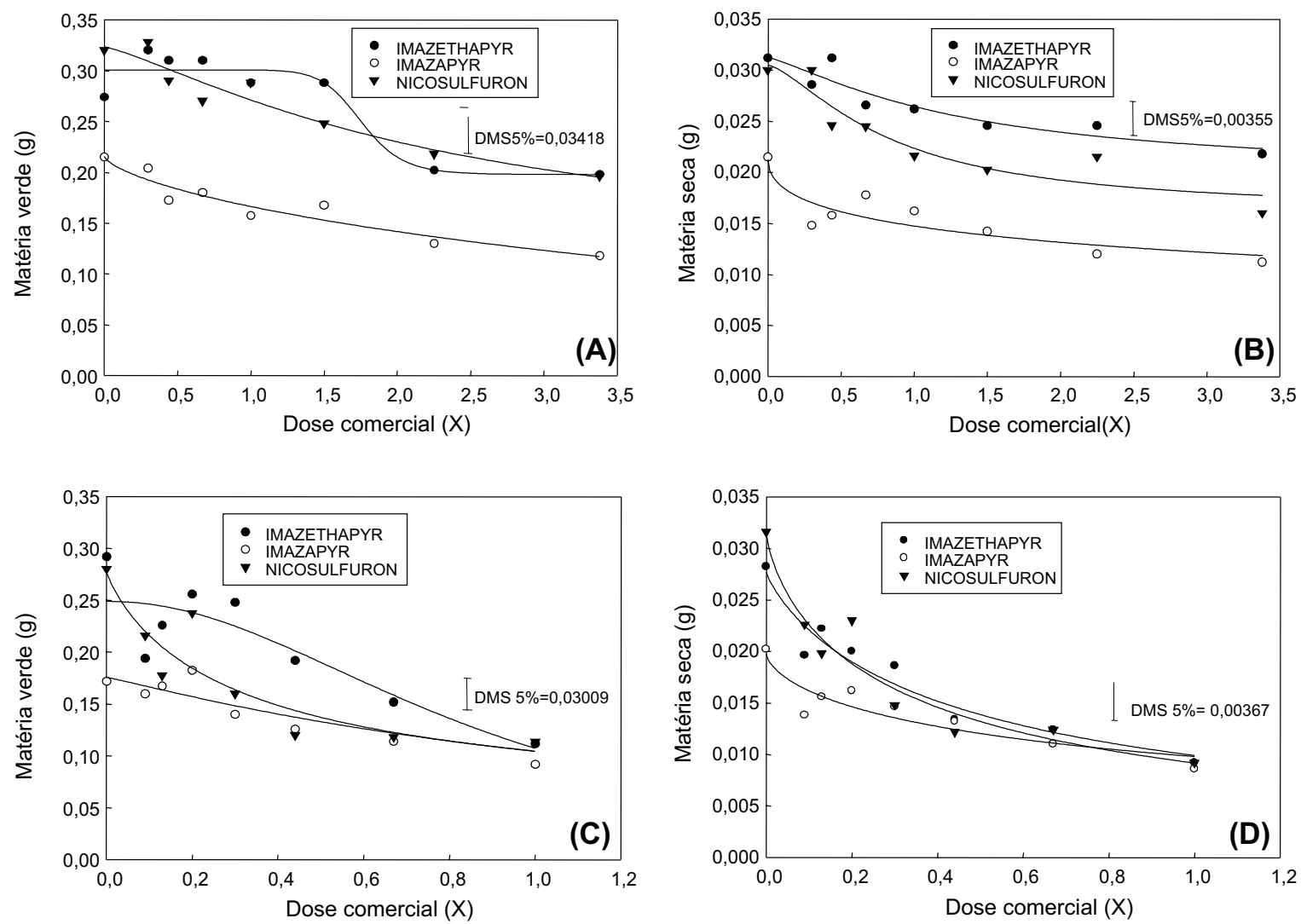

Figura 3 - Matéria verde e seca dos biótipos resistente (A, B) e suscetível (C, D) de E. heterophylla em resposta à imersão com níveis de herbicidas inibidores da ALS

concentrações cerca de dez vezes menores do que a comercial, foram constatados niveis de controle de 76,74 e $48 \%$, aos 5 DAI, respectivamente para fomesafen, lactofen e carfentrazone (Figura 2F). Já para os herbicidas inibidores da ALS, os níveis de controle com a utilização de concentrações tão baixas foram muito inferiores aos obtidos com inibidores da PROTOX, mesmo na avaliação aos 10 DAI (Figura 1F). Niveis tão elevados de controle com doses reduzidas de inibidores da PROTOX são, provavelmente, resultantes do método utilizado, em que ocorre a imersão completa das folhas na solução herbicida, comparativamente ao processo de aspersão, em que gotas do herbicida são depositadas na parte aérea das plantas - com cobertura variável de acordo com a tecnologia de aplicação utilizada. A metodologia de imersão é especialmente vantajosa para herbicidas de contato, como é o caso dos inibidores da PROTOX. A natureza sistêmica dos herbicidas inibidores da ALS (Trezzi \& Vidal, 2001) provavelmente determina niveis de controle mais próximos dos obtidos em aspersões convencionais. Além disso, é possível que avaliações em períodos posteriores a $10 \mathrm{DAI}$, não realizadas no presente experimento, possam resultar em detecção de sintomas mais pronunciados com a utilização de concentração mais baixa de inibidores da ALS.

Considerando os três períodos de avaliação de controle pelos herbicidas inibidores da ALS, não se detectou comportamento geral de superioridade de um herbicida sobre o biótipo $\mathrm{S}$ ou $\mathrm{R}$ (Figura 1). As maiores diferenças de niveis de controle entre os herbicidas foram encontradas nos niveis 2,25X e 3,38X, em que o herbicida imazapyr superou os niveis de controle de plantas $\mathrm{R}$ proporcionados por imazethapyr e nicosulfuron (Figura 1B).

Para os inibidores da PROTOX, o herbicida lactofen destacou-se pela superioridade de controle do biótipo $R$, nas avaliações aos 3 e 5 DAI (Figura 2B, C). Já na avaliação efetuada 
em 1 DAI, lactofen superou os demais inibidores da PROTOX apenas nas concentrações mais elevadas, de 7,58X e 11,39X (Figura 3A). Em relação ao biótipo $\mathrm{S}$, fomesafen controlou mais rapidamente (1 DAI) que os demais herbicidas inibidores da PROTOX, porém os niveis de controle foram similares aos do lactofen nas avaliações mais tardias (3 e 5 DAI). De modo geral, o carfentrazone foi menos eficaz que os herbicidas fomesafen e lactofen para o biótipo $\mathrm{S}$, enquanto o fomesafen foi o menos eficaz em controlar o biótipo $R$.

O emprego da concentração comercial (1X) dos inibidores da ALS reduziu as MV do biótipo S em 33,7, 61,6 e 59,3\%, respectivamente para imazapyr, imazethapyr e nicosulfuron. Contudo, a imersão das folhas do biótipo R na solução com maior concentração dos herbicidas referidos resultou em redução de apenas 45,1 , 27,7 e 38,7\% da MV, em relação à testemunha sem herbicidas (Figura 3A, C). A imersão das folhas do biótipo $\mathrm{S}$ em concentração comercial (1X) de herbicidas inibidores da PROTOX
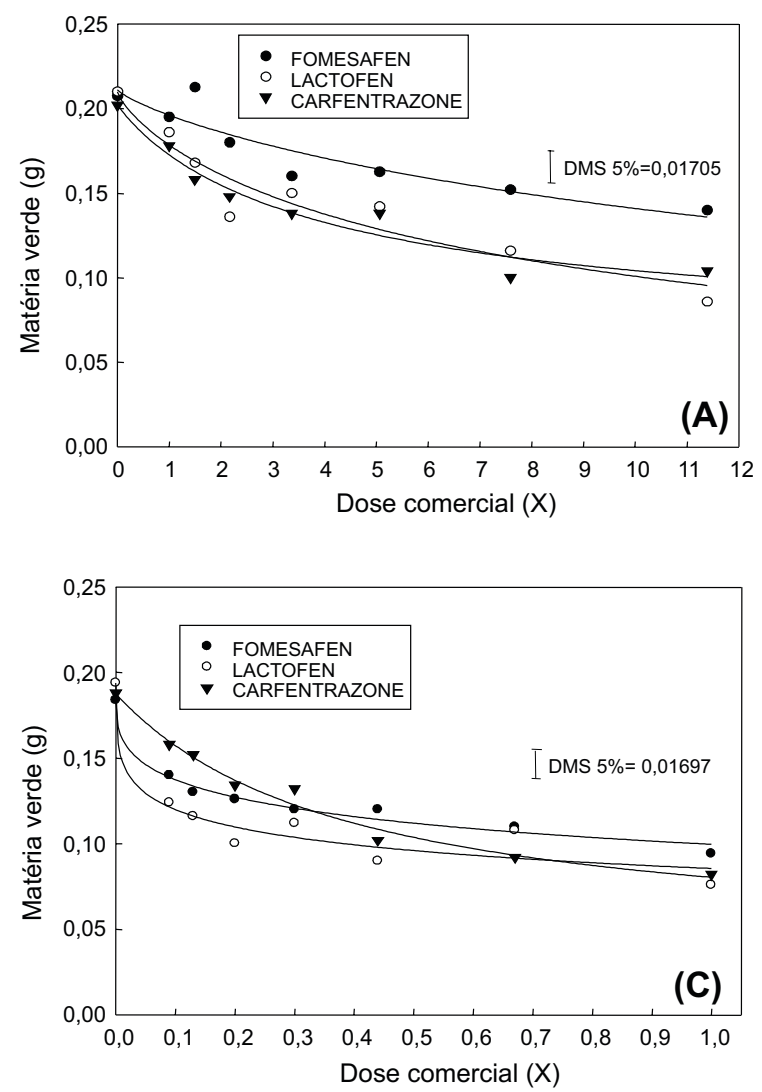

resultou em reduções de MV do biótipo $\mathrm{S}$ de $48,9,60,8$ e $56,4 \%$, respectivamente para fomesafen, lactofen e carfentrazone. Já a mesma concentração resultou em reduções da MV do biótipo R de 6,0, 11,4 e 11,9\%, com a utilização dos mesmos herbicidas (Figura 4A, C). Para os resultados de MS, quando comparadas as maiores doses testadas de inibidores de ALS, tanto para os biótipos S (dose comercial) como para os $R$ ( 3,38 vezes a dose comercial) com as suas testemunhas, encontraram-se reduções de, 67,4 e 30,13\% para o imazethapyr, de 57,43 e $47,90 \%$ para o imazapyr e de $70,9 \%$ e $46,7 \%$ para o nicosulfuron, respectivamente. Os resultados de MV e MS estão de acordo com a avaliação dos niveis de controle, que já haviam demonstrado diferenças muito significativas entre os biótipos R e S.

Os valores de $\mathrm{I}_{50}$, ou seja, os níveis de herbicidas necessários para produzir $50 \%$ de controle ou reduzir $50 \%$ de desenvolvimento, são descritos nas Tabelas 1 e 2. Para os herbicidas inibidores da ALS, observa-se tendência
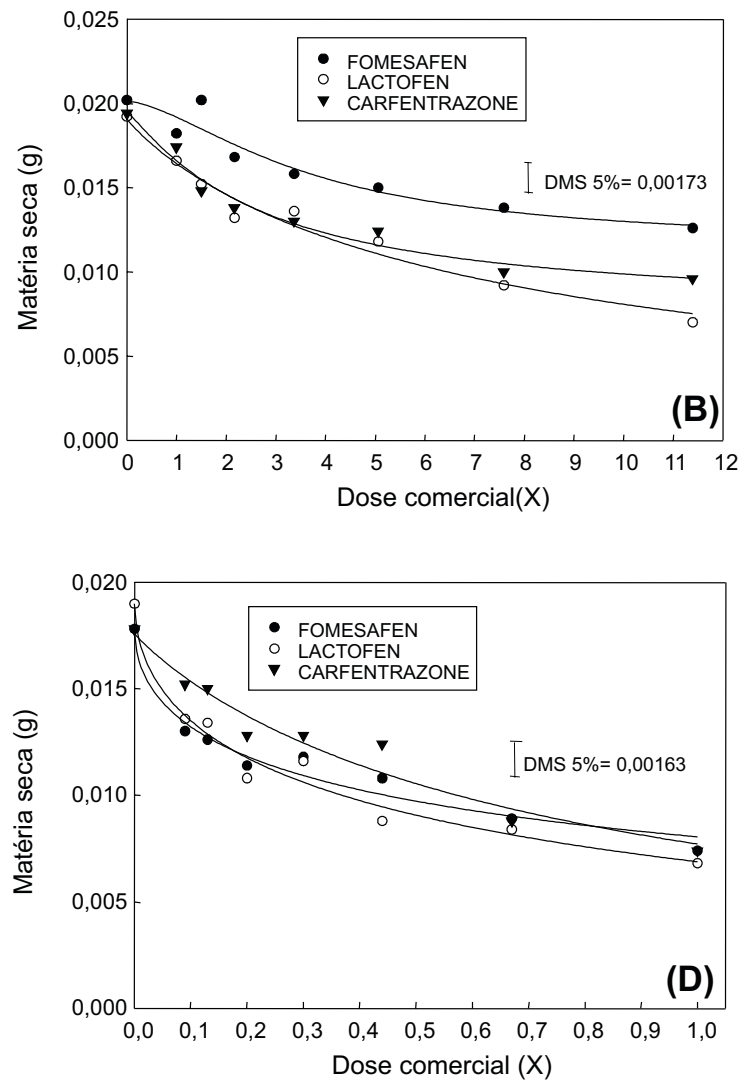

Figura 4 - Matéria verde e seca dos biótipos resistente (A, B) e suscetível (C, D) de E. heterophylla em resposta à imersão com níveis de herbicidas inibidores da PROTOX. 
Tabela 1 - Níveis de imazethapyr, imazapyr e nicosulfuron (inibidores de ALS), necessários para reduzir em 50\% o desenvolvimento dos biótipos resistente e suscetível de E. heterophylla $\left(I_{50}\right)$, e fatores de resistência (FR)

\begin{tabular}{|c|c|c|c|c|c|c|c|c|c|}
\hline \multirow{3}{*}{ Avaliação } & \multicolumn{6}{|c|}{ Valores de $\mathrm{I}_{50} * *$} & & & \\
\hline & \multicolumn{3}{|c|}{ Resistente } & \multicolumn{3}{|c|}{ Suscetível } & \multicolumn{3}{|c|}{$\mathrm{FR}^{* * *}$} \\
\hline & Imazethapyr & Imazapyr & Nicosulfuron & Imazethapyr & Imazapyr & Nicosulfuron & Imazethapyr & Imazapyr & Nicosulfuron \\
\hline Controle 4 DAI* & $1,08^{-/}$ & $49,85^{2 /}$ & $1,50^{-1 /}$ & $0,65^{\frac{1 /}{}}$ & $0,70^{4 /}$ & $0,67^{1 /}$ & 1,65 & 71,21 & 2,23 \\
\hline Controle 7 DAI & $1,04^{\frac{1 /}{}}$ & $2,52^{\frac{3 /}{}}$ & $2,55^{4^{\prime}}$ & $0,63^{3 /}$ & $0,46^{\frac{1}{}}$ & $0,27^{4 /}$ & 1,66 & 5,48 & 9,44 \\
\hline Controle 10 DAI & $46,15^{1 /}$ & $79,07^{1 /}$ & $2,94^{4 /}$ & $0,71^{1 / /}$ & $0,28^{\frac{1}{}}$ & 0,13 & 65,00 & 282,39 & 22,62 \\
\hline Matéria verde & $1,76^{-1}$ & $19,12^{1^{\prime}}$ & $2,40^{\frac{1}{}}$ & $0,88^{\frac{2}{1}}$ & $1,41^{2 /}$ & $0,34^{1 /}$ & 2,00 & 13,56 & 7,06 \\
\hline Matéria seca & $1,32^{\frac{1}{}}$ & $5,52^{\frac{2}{}}$ & $0,84^{1 /}$ & $0,50^{2 /}$ & $0,99^{2^{\prime}}$ & $0,37^{1 /}$ & 2,64 & 5,58 & 2,27 \\
\hline
\end{tabular}

*Dias após imersão no tratamento; ** nível de herbicida que produz $50 \%$ de controle $\left(\mathrm{I}_{50}\right)$; ${ }^{* * *} \mathrm{FR}=\mathrm{I}_{50}$ resistente/ $\mathrm{I}_{50}$ suscetível. ${ }^{1 /}$ Modelo logístico de quatro parâmetros. ${ }^{2 /}$ Modelo logístico de três parâmetros. ${ }^{3 /}$ Modelo sigmoidal de quatro parâmetros. ${ }^{4 /}$ Modelo sigmoidal de três parâmetros

Tabela 2 - Níveis de fomesafen, lactofen e carfentrazone (inibidores de PROTOX), necessários para reduzir em 50\% o desenvolvimento dos biótipos resistente e suscetível de E. heterophylla $\left(\mathrm{I}_{50}\right)$, e fatores de resistência $(\mathrm{FR})$

\begin{tabular}{|c|c|c|c|c|c|c|c|c|c|}
\hline \multirow{3}{*}{ Avaliação } & \multicolumn{6}{|c|}{ Valores de $\mathrm{I}_{50}{ }^{* *}$} & & & \\
\hline & \multicolumn{3}{|c|}{ Resistente } & \multicolumn{3}{|c|}{ Suscetível } & \multicolumn{3}{|c|}{$\mathrm{FR}^{* * * *}$} \\
\hline & Fomesafen & Lactofen & Carfentrazone & Fomesafen & Lactofen & Carfentrazone & Fomesafen & Lactofen & Carfentrazone \\
\hline Controle 1 DAI* & $3,05^{\frac{2}{}}$ & $7,58^{1 /}$ & $32,75^{\frac{1}{1}}$ & $0,12^{\frac{1}{}}$ & $0,94^{\frac{1}{\prime}}$ & $0,16^{-/}$ & 25,42 & 8,06 & 204,69 \\
\hline Controle 3 DAI & $8,84^{1 /}$ & $2,70^{-1 /}$ & $4,89^{1 /}$ & $0,07^{2} !$ & $0,09^{\frac{1 /}{}}$ & $1,77^{1 /}$ & 126,29 & 30,00 & 2,76 \\
\hline Controle 5 DAI & $2,25^{1^{\prime}}$ & $3,94^{1 /}$ & $1,77^{1 /}$ & $0,06^{4 /}$ & $0,04^{\frac{1 /}{\prime}}$ & $0,19^{\frac{1 /}{}}$ & 37,50 & 98,50 & 9,32 \\
\hline Matéria verde & $23,53^{2 /}$ & $9,04^{2 /}$ & $4,07^{1 /}$ & $1,51^{2 !}$ & $0,47^{2 !}$ & $0,38^{\frac{1 /}{}}$ & 15,58 & 19,23 & 10,71 \\
\hline Matéria seca & $3,66^{-/}$ & $7,17^{2 /}$ & $2,71^{1 /}$ & $0,72^{2 !}$ & $0,85^{\frac{1 /}{}}$ & $0,85^{2 \prime}$ & 5,08 & 8,44 & 3,19 \\
\hline
\end{tabular}

* Dias após imersão no tratamento; ** nível de herbicida que produz $50 \%$ de controle $\left(\mathrm{I}_{50}\right) ; * * * \mathrm{FR}=\mathrm{I}_{50}$ resistente/ $\mathrm{I}_{50}$ suscetível. ${ }^{1 /}$ Modelo logístico de quatro parâmetros. ${ }^{2 /}$ Modelo logístico de três parâmetros. ${ }^{3 /}$ Modelo sigmoidal de quatro parâmetros. ${ }^{4 /}$ Modelo sigmoidal de três parâmetros

de elevação dos valores de $\mathrm{I}_{50}$ do biótipo $\mathrm{R}$ com o decorrer do periodo de avaliação, com exceção do herbicida imazapyr (Tabela 1). Ao contrário, para herbicidas com esse mecanismo de ação, os valores de $\mathrm{I}_{50}$ do biótipo $\mathrm{S}$ tendem à diminuição, com exceção de imazethapyr (Tabela 1). Isso resulta em valores de fator de resistência (FR) maiores com o avanço dos períodos de avaliação de controle para os herbicidas inibidores da ALS (Tabela 1), já que FR representa a razão entre $\mathrm{I}_{50}$ 's dos biótipos $\mathrm{R}$ e $\mathrm{S}$. Isso significa que há recuperação das plantas $\mathrm{R}$ com o decorrer do tempo, após a aplicação dos herbicidas inibidores da ALS, ao contrário do que ocorre com as plantas S.

Para os herbicidas inibidores da PROTOX, os valores de $\mathrm{I}_{50}$ para controle visual não obedecem a um padrão regular de aumento ou diminuição em função do período de avaliação, tanto para os biótipos $\mathrm{R}$ quanto para $\mathrm{S}$ (Tabela 2), assim como os valores de FR (Tabela 2).

Os valores de FR da variável controle através de inibidores da ALS variaram entre 1,6 ae282,4. Essa variável, quando determinada pelo controle por inibidores da PROTOX, variou entre 2,8 e 204,7. Os valores de FR, obtidos pela MV, variaram entre 2 e 13,6 e, pela MS, entre 2,3 e 5,6, para inibidores da ALS. Para inibidores da PROTOX, os valores de FR foram sensivelmente maiores, variando entre 10,7 e 19,2 (MV) e entre 3,2 e 8,4 (MS).

Os valores de FR são variáveis de acordo com o herbicida utilizado, biótipo, condições de ambiente e variável mensurada, o que leva a encontrar na literatura valores distintos de FR em ensaios com EPHHL, tanto para inibidores da ALS quanto para inibidores da PROTOX. Testando o efeito dos herbicidas chlorimuronethyl e imazethapyr, inibidores da ALS, sobre a matéria seca de EPHHL, em ambiente de casa de vegetação, Gelmini et al. (2001) encontraram FRs de 22 e 23, respectivamente. Em ensaio de resistência de EPHHL a inibidores da PROTOX em casa de vegetação em Pato Branco (PR), Trezzi et al. (2009) encontraram FRs de 1,4 a 2,1 para a variável controle pelo herbicida fomesafen. 
Valores de FR superiores a 1,0 são indicativos de resistência. Os resultados apresentados neste trabalho demonstram a existência de resistência cruzada do biótipo de EPHHL tanto a inibidores da ALS quanto a inibidores da PROTOX. No entanto, segundo os Critérios Oficiais para a Confirmação de Resistência no Brasil (Gazziero et al., 2008), experimentos em ambiente controlado e em campo devem ser realizados para efetivamente conferir a uma população suspeita o status de R ou S.

Os resultados encontrados respaldam a técnica da imersão foliar como adequada para a discriminação de biótipos com resistência múltipla a inibidores da ALS e PROTOX e dos biótipos S. A técnica demonstrou ser rápida o suficiente para a detecção precoce da resistência, o que possibilita, provavelmente, a adoção de medidas em campo ainda na mesma safra para contornar o problema. O teste ainda apresenta a vantagem de permitir a confirmação da resistência na própria propriedade dos agricultores, pois a dose comercial dos herbicidas se revelou altamente discriminadora.

\section{LITERATURA CITADA}

BOUTSALIS, P. Syngenta Quick-Test: a rapid whole-plant test for herbicide resistance. Weed Technol., v. 15, n. 2, p. 257-263, 2001.

CARVALHO, S. J. P. et al. Identificação de biótipos de Bidens spp. resistentes aos inibidores da ALS através de teste germinativo. Planta Daninha, v. 22, n. 3, p. 411-417, 2004.

CHRISTOFFOLETI, P. J.; LÓPEZ-OVEJERO, R. F. Resistência de plantas daninhas a herbicidas: definições, bases e situação no Brasil e no mundo. In: CHRISTOFFOLETI, P J. (Orgs.). Aspectos de resistência de plantas daninhas a herbicidas. 3.ed. Piracicaba: HRAC-BR, 2008. p. 3-26.

CONCENÇO, G. et al. Método rápido para detecção de resistência de capim-arroz (Echinochloa spp.) ao quinclorac. Planta Daninha, v 26, n. 2, p. 429-437, 2008.

FRANS, R. et al. Experimental design and techniques for measuring and plant responses to weed control practices. In: CAMPER, N. D. Research methods in weed science. 3.ed. Champaign: Southern Weed Science Society, 1986. p.29-46.

GAZZIERO, D. L. P. et al. Critérios para relatos oficiais estatísticos de biótipos de plantas daninhas resistentes a herbicidas. Sete Lagoas: Sociedade Brasileira da Ciência das Planas Daninhas; Campinas: Associação Brasileira de Ação à Resistência de Plantas aos Herbicidas no Brasil, 2008. 22 p.
GELMINI, G. A. et al. Resistência de biótipos de Euphorbia heterophylla L. aos herbicidas inibidores da enzima ALS utilizados na cultura de soja. Bragantia, v. 60, n. 2, p. 93-99, 2001.

HEAP, I. International survey of herbicide-resistant weeds. 2011. Disponível em: <http://www.weedscience.com> Acesso em: 20 de julho de 2011.

KIM, D. S.; RICHES, C.; VALVERDE, B. Rapid detection of propanil and fenoxaprop resistance in Echinochloa colona. Weed Sci., v. 48, n. 6, p. 695-700, 2000.

MONQUERO, P. A.; CHRISTOFFOLETI, P. J. Manejo de populações de plantas daninhas resistentes aos herbicidas inibidores da acetolactato sintase. Planta Daninha, v. 19, n. 1, p. 67-74, 2001a.

MONQUERO, P. A.; CHRISTOFFOLETI, P. J. Bioensaio rápido de determinação da sensibilidade da acetolactato sintase (ALS) a herbicidas inibidores. Sci. Agric., v. 58, n. 1, p. 193-196, 2001b.

NORSWORTHY, J. K.; TALBERT, R. E.; HOAGLAND, R. E. Chlorophyll fluorescence for rapid detection of propanilresistant barnyardgrass (Echinochloa crus-galli). Weed Sci., v. 46, n. 2 , p. $163-169,1998$.

PORTES, E. D. S. Eleusine indica (L.) Gaertn. resistente a alguns inibidores de ACCase e novo método para diagnóstico de plantas daninhas resistentes aos herbicidas. 2005. 85 f. (Programa de Pós-Graduação em Fitotecnia) - Universidade Federal do Rio Grande do Sul, Porto Alegre, 2005.

RICHTER, J.; POWLES. S. B. Pollen expression of herbicide target site resistance genes in annual ryegrass (Lolium rigidum). Plant Physiol., v. 102, n. 3, p. 1037-1041, 1993.

SAARI, L. L.; COTTERMAN, J. C.; THILL, D. C. Resistance to acetolactate synthase inhibiting herbicides. In: POWLES, S. B.; HOLTUM, J. A. M. Herbicide resistance in plants: biology and biochemistry. Boca Raton: CRC Press, 1994. $353 \mathrm{p}$.

SEEFELDT, S. S.; JENSEN, J. E.; FUERST, E. P. Log logistic analysis of herbicide dose-response relationships. Weed Technol., v. 9, n. 2, p. 218-227, 1995.

TREZZI, M. M. ; VIDAL, R. A. Herbicidas inibidores da ALS. In: VIDAL, R. A.; MEROTTO JR., A. (Orgs.). Herbicidologia. Porto Alegre: Edição dos Autores, 2001.p. 25-36

TREZZI, M. M. et al. Multiple resistance of acetolactate synthase and protoporphyrinogen oxidase inhibitors in Euphorbia heterophylla biotypes. J. Environ. Sci. Health Part B, v. 40, n. 1, p. 101-109, 2005.

Planta Daninha, Viçosa-MG, v. 29, n. 4, p. 901-912, 2011 
TREZZI, M. M. et al. Local de absorção de fomesafen como mecanismo de resistência em biótipo de Euphorbia heterophylla resistente aos inibidores da PROTOX

Planta Daninha, v. 27, n. 1, p. 139-148, 2009.

VIDAL, R. A.; MEROTTO JR., A. Resistência de amendoimbravo (Euphorbia heterophylla L.) aos herbicidas inibidores da enzima acetolactato sintase. Planta Daninha, v. 17, n. 3 , p. 367-374, 1999.
VIDAL, R. A. et al. UFRGS-SUPER-QUICK herbicide resistance diagnostic test. In: MEETING OF THE WSSA, 2005, Honolulu. Abstracts. Honolulu: WSSA, 2005. p. 68 .

VIDAL, R. A.; LAMEGO, F. P.; TREZZI, M. M. Diagnóstico da resistência aos herbicidas em plantas daninhas. Planta Daninha, v. 24, n. 3, p. 597-604, 2006. 